\title{
Dietary fatty acids, cholesterol, and behavior in the rat
}

\author{
B. MICHAEL THORNE, RENA POPMA, and WERNER ESSIG \\ Mississippi State University, Mississippi State, Mississippi
}

\begin{abstract}
The behavioral effects and effects on serum cholesterol of diets containing different fats were studied in Long-Evans hooded rats. Adult males were fed one of four diets: AIN-76A, Group CON; AIN-76A plus 20\% safflower oil, Group POLY; AIN-76A plus 20\% coconut oil, Group SAT; AIN-76A plus $20 \%$ olive oil, Group MONO. After 29 days, the rats were tested for irritability toward handling, open-field activity, passive-avoidance learning on a modified apparatus, and visual discrimination learning with reversal. The rats in Group POLY had lower total-cholesterol-to-HDLcholesterol ratios than did animals in any other group. Except for shorter latencies by Group MONO rats to leave the platform on the passive-avoidance training trials, no behavioral differences were observed.
\end{abstract}

Studies indicate that diet can influence behavior (e.g., Wurtman, 1982). Manipulating dietary fat can cause behavioral changes (e.g., Coscina, Yehuda, Dixon, Kish, \& Leprohon-Greenwood, 1986; Lamptey \& Walker, 1976; Yehuda, Leprohon-Greenwood, Dixon, \& Coscina, 1986), possibly by causing changes in cholesterol level and neuronal membrane fluidity (Yehuda, 1987).

Lamptey and Walker (1976) found that the offspring of female rats fed soybean oil showed superior learning compared with rats whose dams were fed a safflower oil supplement. Also, rats fed a diet for 3 weeks containing $20 \%$ polyunsaturated fat (soybean oil) performed better on a water maze than rats fed either a diet with $20 \%$ saturated fat (lard) or a Purina Lab Chow diet $(4.5 \%$ mixed fats) (Coscina et al., 1986). Improved performance was not attributable to increased activity. Using the same diets, Yehuda et al. (1986) found that albino rats fed soybean oil had increased pain thresholds, and that both albino and hooded rats fed soybean oil had increased resistance to hypothermia. Yehuda and Carasso (1987) compared rats fed diets containing soybean oil, sunflower oil, or lard with control animals and found that the soybean oil diet improved learning ability and caused a pain threshold increase.

Yehuda (1987) proposed that dietary manipulation may alter behavior by changing the neuronal membrane lipid composition. In a study of neuronal membrane cholesterol content and learning ability, Kessler and Yehuda (1985) found that learning per se induced significant cholesterol changes in the hippocampus and the cortex of rats. $\mathrm{Re}$ -

This research was supported in part by an institutional grant from the National Science Foundation to Mississippi State University. The authors would also like to thank Joe Washington for help in the data collection. Reprint requests should be sent to Mike Thorne, Department of Psychology, P.O. Drawer 6161, Mississippi State University, Mississippi State, MS 39762. gional cholesterol level was decreased, with a corresponding increase in membrane fluidity.

Dietary fats can also affect serum cholesterol levels. Early research in humans found that polyunsaturated fats were superior to monounsaturated fats in reducing cholesterol levels, while saturated fats raised cholesterol levels (e.g., Keys, Anderson, \& Grande, 1957). Some recent studies on humans have suggested that a high monosaturated fat diet is superior to either a high polyunsaturated fat diet or to a low-fat diet in lowering serum cholesterol (e.g., Grundy, 1986). Recent studies of dietary fat and rats' serum cholesterol agree with the early human studies; polyunsaturated fats lower cholesterol relative to monounsaturated or saturated fats (e.g., Lim, Oh, \& Han, 1985).

The purpose of this study was to investigate further the relationship between dietary fat and behavior. Four groups of rats were fed either a standard base diet or the base diet with added safflower oil, olive oil, or coconut oil. Safflower oil and olive oil were chosen because they contain the highest percentages of polyunsaturated and monounsaturated fat, respectively, of any commercially available oils. Coconut oil was used as the saturated fat since it is a vegetable oil rather than an animal fat. The dependent variables assessed activity, aggression, and learning ability. As a further check on the dietary manipulations, serum cholesterol was measured at the study's conclusion.

\section{METHOD}

\section{Subjects}

Thirty-six adult male Long-Evans hooded rats, approximately 4.5-6 months old, were assigned by weight to four groups of equal numbers. The rats were fed either the base diet (AIN-76A, American Institute of Nutrition, 1977) or the AIN-76A diet with $20 \%$ added fat. The caloric densities of the three added-fat diets were identical, while the AIN$76 \mathrm{~A}$ diet with only $5 \%$ fat (corn oil) had fewer calories.

The rats were bred and weaned at intervals, so that the experiment was run in three replications of 12 rats each ( 3 animals in each dietary group). Three animals died during the study, resulting in the following 
group totals: AIN-76A diet group (Group CON), 8; safflower oil diet group (Group POLY), 9; coconut oil diet group (Group SAT), 9; and olive oil diet group (Group MONO), 7.

All rats were housed singly, in stainless steel cages. Food and water were available ad lib, and 12:12-h light:dark (8 a.m. on, 8 p.m. off) housing conditions were maintained. All testing occurred during the light portion of the cycle.

\section{Apparatus}

Open-field activity was assessed in a $76.2 \times 76.2 \times 25.4 \mathrm{~cm}$ box painted flat black with a floor divided by white lines into 25 equal squares. The top was covered with wire mesh.

Passive-avoidance training and testing were performed in an apparatus similar to the one shown in Thorne and Rowles (1988). The apparatus consisted of two discriminably different rear chambers joined to a lighted outer platform. Both posterior chambers had a grid floor for shock administration. Guillotine doors separated the back chambers from the platform.

The visual discrimination apparatus was a Thompson-Bryant twochoice box (Thompson \& Bryant, 1955) without a choice chamber. The combination startbox/choice chamber had a grid floor for applying footshock. A Plexiglas door separated the start area from a 7.6-cm grid in front of two openings to the goalbox. Each opening was blocked by a $10.2 \times 16.5 \mathrm{~cm}$ stimulus card made from either black or white construction paper pasted on heavy posterboard. The cards were inserted into clear plastic sleeves, which were washed with water after each animal's testing. Painted gray cards were used for preliminary training trials.

\section{Procedure}

The rats were weighed at the beginning of the study, weekly thereafter, and at sacrifice. Food intake was assessed weekly.

After 29 days on the diets, for 4 consecutive days, each animal and its cage were carried to a table. There, each animal was given a 1-min test in or near the homecage to assess reactions to perioral stimulation and flank tap with a pencil, to capture, and to being held. Vocalizations during the test and all other components were rated on a scale from 0-3. Daily scores were summed and used as each subject's handling score. Interobserver reliability measurements revealed significant agreement $\left(r_{s}=0.59, p<.01\right)$

After the handling test, each subject was placed onto the center of the open-field apparatus. The number of squares crossed and the number of rearings were recorded for $1 \mathrm{~min}$. The apparatus was cleaned with water after each subject was removed, and the testing order was changed daily. Interobserver reliability was high [horizontal activity, $r(42)=0.99, p<.01$; rearing, $r(42)=0.98, p<.01$ ].

Next, each animal received four acquisition trials on the modified passive-avoidance apparatus. On each trial, a rat was placed on the outer platform and its latency to enter the open chamber was recorded. Only one chamber was open on any acquisition trial, and the open chamber's location was alternated. For half the rats, Chamber A was on the left and Chamber B on the right. The chambers were reversed for the other half. During a 1-min intertrial interval, the rats were placed into their homecages, located near the apparatus.

After the fourth trial, the entrance to the chamber chosen was blocked, and a 1-mA, 3-sec footshock was administered. After $24 \mathrm{~h}$, the rat was placed on the outer platform with both chambers open. Entrance latency and the chamber entered were recorded. The postshock trial ended either upon chamber entry or after $5 \mathrm{~min}$.

On the day after the postshock trial, training began on a visual discrimination task. Each rat received five preliminary training trials to learn to push aside a gray card to enter the goalbox. Position preference was noted, and the positive card was initially placed on the animal's preferred side.

On the day after preliminary training, training began on the whiteblack discrimination. On each trial, the rat was first placed into the startbox. After $5 \mathrm{sec}$, the Plexiglas door was raised, and the animal was given approximately $15 \mathrm{sec}$ to make an unforced choice. For half the subjects, the initial discrimination was white-positive, black-negative; for the other half, it was the opposite. Choice of the negative card led to mild footshock, and each trial ended when the animal entered the goalbox by pushing the positive card. The position of the cards, but not the plastic sleeves, varied according to a modified random sequence. The rats were given 10 trials/day, with a 1-min intertrial interval. Training continued until the rat reached a criterion of nine correct responses in 1 day. Learning ability was assessed by the number of errors an animal made prior to reaching criterion.

After the criterion was reached on the original discrimination, the previously positive card became negative and vice versa. Training on the reversal problem continued until all animals attained criterion on at least one reversal. The rats that reached criterion relatively quickly on the first reversal were trained on subsequent reversals.

Finally, all the rats were deeply anesthetized with sodium pentobarbital and weighed, and a blood sample was taken by cardiac puncture. The blood samples were analyzed for total cholesterol (TC) and for highdensity lipoprotein cholesterol (HDL), using a Kodak Ektachem Analyzer (Allain, Poon, Chan, Richmond, \& Fu, 1974; Finley, Shifman, Williams, \& Lichti, 1978).

\section{RESULTS}

\section{Body Weight and Food Intake}

All rats gained weight over time, and a two-way ANOVA (dietary group vs. measurement period) revealed a significant main effect for measurement period $[F(7,203)=58.63, p<.0001]$, while neither the groups main effect nor the interaction were significant. Weight at sacrifice was significantly correlated with total cholesterol levels $[r(30)=0.39, p<.05]$.

When food intake was adjusted for dietary caloric differences, one-way ANOVAs indicated there were no significant between-groups differences at any measurement period. Since Group CON rats consumed more of the AIN-76A diet in order to equate caloric intake with animals in the added-fat groups, added-fat animals received less of the vitamin-and-mineral mix. However, the AIN-76A diet exceeds the daily nutrient requirement for vitamins and minerals for a rat at maintenance. Since no animal showed evidence of a vitamin or mineral deficiency, the exposure levels of the added-fat animals must have been adequate.

\section{Irritability toward Handling and Open-Field Activity}

A Kruskal-Wallis test revealed no significant betweengroups differences on the handling test. One-way ANOVAs revealed no significant differences between groups for either horizontal activity or rearing in the open field.

\section{Passive Avoidance}

Training trials. There were significant between-groups differences on latency to leave the outer platform on the third of the four passive-avoidance training trials (KruskalWallis one-way ANOVA by ranks, $H=8.15, p<.05$ ). Groups POLY and SAT took significantly longer to leave the platform than did Group MONO (POLY vs. MONO: Mann-Whitney $U=6, p<.05$; SAT vs. MONO: $U=$ $8, p<.05)$.

The between-groups latency differences for the second and fourth trials approached statistical significance 
$(H=7.79, p<.06 ; H=7.59, p<.06$, respectively). Group CON rats took longer to leave the platform than did Group MONO rats on the second trial $(U=6$, $p<.05$ ), and rats in Groups CON and POLY took longer to leave than did Group MONO rats on the fourth trial ( $U=8, p<.05 ; U=3, p<.01$, respectively).

A test using dependent $t$ scores to compare first-trial latency with fourth-trial latency provided no evidence for habituation.

Postshock trial. One rat from Group CON and 1 from Group POLY did not leave the platform on the fourth training trial, and both were excluded from further passive avoidance. All except 1 rat stayed on the outer platform for $5 \mathrm{~min}$ on the postshock trial, and there were no between-groups differences. A Group MONO rat entered the chamber where it had not received shock.

\section{Discrimination Reversal}

One-way ANOVAs revealed no significant betweengroups differences in the number of errors prior to criterion on either the initial discrimination or the first reversal. The groups did not differ on the number of completed reversal problems.

\section{Serum Cholesterol}

The group means and standard errors for TC, HDL, and the TC/HDL ratios are shown in Table 1 . The summarized results of significance testing (one-way ANOVAs, followed by Fisher $L S D$ tests) are shown below each table column.

\section{DISCUSSION}

The added-fat diets used in this study did not affect weight change, handling characteristics, open-field activity, or learning ability. Failure to find an increase in activity after diets containing either added polyunsaturated or saturated fats agrees with previous reports (Coscina et al., 1986; Yehuda et al., 1986).

We found no evidence for improved learning after added safflower oil, in contrast to the finding of superior water-maze learning in rats fed diets with added soybean oil (Coscina et al., 1986). Although soybean oil is considered a polyunsaturated fat, it contains only slightly more polyunsaturated fat than monounsaturated fat. Added soybean oil diets have often led to superior learning in rats, relative to animals fed diets containing other added fats (e.g., safflower oil, Lamptey \& Walker,

Table 1

Serum Cholesterol Results

\begin{tabular}{|c|c|c|c|c|c|c|c|}
\hline \multirow[b]{2}{*}{ Group } & \multirow[b]{2}{*}{$n$} & \multicolumn{2}{|c|}{ TC } & \multicolumn{2}{|c|}{ HDL } & \multicolumn{2}{|c|}{ TC/HDL } \\
\hline & & $M$ & $S E M$ & $M$ & $S E M$ & $M$ & $S E M$ \\
\hline $\mathrm{CON}$ & 8 & 123.00 & 7.83 & 57.00 & 6.51 & 2.24 & 0.14 \\
\hline POLY & 9 & $90.44 *$ & 6.18 & 51.44 & 3.18 & $1.76 \S$ & 0.06 \\
\hline SAT & 9 & $86.78^{*}$ & 5.04 & $40.11 \dagger$ & 2.57 & 2.18 & 0.10 \\
\hline MONO & 7 & $82.71 *$ & 12.95 & $34.00 \ddagger$ & 3.55 & 2.38 & 0.15 \\
\hline
\end{tabular}

Note-Mean \pm SEMs (in $\mathrm{mg} / \mathrm{dl}$ ) are for total cholesterol (TC), highdensity lipoprotein cholesterol (HDL), and TC/HDL ratios by dietary group. *Significantly different from Group CON, $p<.01$. $\nmid$ Significantly different from Group CON, $p<.01$. ‡Significantly different from Groups CON and POLY, $p<.01$. §Significantly different from Groups CON, SAT, and MONO, $p<.01$.
1976; sunflower oil or lard, Yehuda \& Carasso, 1987). Thus, the present study supports Yehuda and Carasso's conclusion that the level of polyunsaturated fat in the diet is not simply related to improved learning ability.

Safflower oil and soybean oil differ in essential fatty acid composition; safflower oil is deficient in linolenic acid, but soybean oil is deficient in linoleic acid (Lamptey \& Walker, 1976). Thus, the beneficial effect on rat learning seen in several studies after soybean oil exposure may be due to its essential fatty acid composition rather than to its polyunsaturated/saturated fat ratio.

In the modified passive-avoidance task, the rats in Group MONO left the outer platform more quickly than did the rats on the other diets. The differences were not attributable to increased activity levels, since there were no group differences on the open-field test. One speculation for further testing is that the added olive oil increased the light sensitivity of the rats, which made the light over the outer platform more aversive.

Like the rats on the other added-fat diets, Group POLY rats had significantly lower total cholesterol than did Group CON rats, despite the fact that the AIN-76A diet is an extremely low-fat diet with only $5 \%$ fat from corn oil. Unlike the diets containing either olive oil or coconut oil, the safflower oil diet did not lower HDL cholesterol, resulting in rats with the lowest TC/HDL ratios. The beneficial effect of safflower oil on rats' serum cholesterol agrees with other animal studies (e.g., Lim et al., 1985) and with early studies on humans (Keys et al., 1957), but it disagrees with reports of the superiority of monounsaturated fat over polyunsaturated fat in reducing total cholesterol while raising (or maintaining) HDL levels (e.g., Grundy, 1986).

Despite the clear reductions in serum cholesterol in the added-fat groups, the only group behavioral difference was a decrease in exit latencies in Group MONO on the passive-avoidance task; learning ability was not enhanced. An increase in cholesterol level causes a decrease in membrane fluidity (Houslay \& Stanley, 1982). Also, learning itself caused a decrease in hippocampal and cortical cholesterol levels, with an corresponding regional increase in membrane fluidity (Kessler \& Yehuda, 1985). Thus, the present results suggest either that lowering serum cholesterol does not induce the neural cholesterol changes necessary for enhanced learning, or that the learning tasks used in this study were not sensitive enough to detect learning improvements. Although the passive-avoidance task and the initial visual discrimination task are relatively simple, the reversal problem is quite difficult, with animals almost always requiring at least twice as many errors to attain a criterion of $90 \%$ correct.

Future studies might include larger samples, higher dietary fat content, and more sensitive learning measures. In addition, a soybean oil diet should be included in any study of behavioral changes, and physiological measures should include an assay of regional brain cholesterol.

\section{REFERENCES}

Allain, C. C., Poon, L. S., Chan, C. S. G., Richmond, W., \& Fu, P. C. (1974). Enzymatic determination of total cholesterol in serum. Clinical Chemistry, 20, 470-475.

American Institute of Nutrution (1977). Ad Hoc Committee on Standards for Nutritional Studies. Report of the committee. Journal of Nutrition, 107, 1340-1348.

Coscina, D. V., Yehuda, S., Dixon, L. M., Kish, S. J., \& LeProhonGREENWOOD, C. E. (1986). Learning is improved by a soybean oil diet in rats. Life Sciences, 38, 1789-1794.

Finley, P. R., Shifman, R. B., Williams, R. J., \& Lichti, D. A. (1978). Cholesterol in high-density lipoprotein: Use of $\mathrm{Mg}^{2}+/$ dextran sulfate in its enzyme determination. Clinical Chemistry, 24, 931-933.

GRUNDY, S. M. (1986). Comparison of monounsaturated fatty acids and carbohydrates for lowering plasma cholesterol. New England Journal of Medicine, 314, 745-748.

Houslay, M. D., \& Stanley, K. K. (1982). Dynamics of biological membranes. New York: Wiley.

KesSLER, R. A., \& YeHUdA, S. (1985). Learning-induced changes in brain membrane cholesterol and fluidity: Implication for brain aging. International Journal of Neuroscience, 28, 73-82. 
Keys, A., Anderson, J. T., \& Grande, F. (1957). Prediction of serumcholesterol responses of man to changes in fats in the diet. Lancet, 2, 959-966.

LAMPTEY, M. S., \& WALKeR, B. L. (1976). A possible essential role for dietary linolenic acid in the development of the young rat. Journal of Nutrition, 106, 86-93.

LiM, H. S., OH, S. H., \& HaN, I. K. (1985). Effects of dietary polyunsaturated and saturated (P/S) fat ratio on plasma cholesterol and lipoprotein in rats. Korean Journal of Animal Science, 27, 535-539.

ThOMPSON, R., \& BRYANT, J. H. (1955). Memory as affected by activity of the relevant receptor. Psychological Reports, 1, 393-400.

ThorNe, B. M., \& Rowles, J. S. (1988). Memory deficit in passiveavoidance learning in bulbectomized Long-Evans hooded rats. Physiology \& Behavior, 44, 339-345.

Wurtman, R. J. (1982). Nutrients that modify brain function. Scientific American, 246, 42-59.
YeHUDA, S. (1987). Nutrients, brain biochemistry, and behavior: A possible role for the neuronal membrane. International Journal of Neuroscience, 35, 21-36.

Yehuda, S., \& CARASSo, R. L. (1987). Effects of dietary fats on learning, pain threshold, thermoregulation and motor activity in rats: Interaction with the length of feeding period. International Journal of Neuroscience, 32, 919-925.

Yehuda, S., Leprohon-Greenwood, C. E., Dixon, L. M., \& Coscina, D. V. (1986). Effects of dietary fat on pain threshold, thermoregulation, and motor activity in rats. Pharmacology, Biochemistry \& Behavior, 24, 1775-1777.

(Manuscript received August 14, 1989.)

\title{
Notices and Announcements
}

\author{
International Society for Research on Aggression \\ IX Biennial World Meeting \\ Banff, Alberta, Canada \\ June 12-17, 1990
}

The IX Biennial World Meeting of the International Society for Research on Agression will be held in Banff, Alberta, Canada, on June 12-17, 1990.

For further information, contact Gordon W. Russell, Department of Psychology, University of Lethbridge, Lethbridge, Alberta T1K 3M4, Canada. 